\title{
A Numerical and Analytical Study of Vortex Rings With Swirl
}

\author{
Alexander Lifschitz \\ Mathematics Dept. \\ University of Illinois \\ Chicago, IL 60607, USA
}

\author{
W. Henry Suters \\ Mathematics Dept. \\ Carson-Newman College \\ Jefferson City, TN 37760, USA
}

\author{
J. Thomas Beale \\ Mathematics Dept. \\ Duke University \\ Durham, NC 27708, USA
}

\begin{abstract}
We study the growth of disturbances to vortex rings with swirl, which are exact solutions of the Euler equations of inviscid flow, using two contrasting methods. The motion of the perturbed vortex rings can be regarded as a prototype for the inviscid dynamics of vortex structures in 3D. Exact rings with swirl are computed as steady, axisymmetric flows using a variational method. Asymptotic analysis in the short wave limit, similar to geometric optics, leads to ordinary differential equations for perturbations along particle paths. These ODE's can be solved for the rings of interest, yielding predicted maximum growth rates for small disturbances. These rates are compared with the direct simulation of sample disturbances using a $3 \mathrm{D}$ vortex method to calculate the evolution according to the Euler equations.
\end{abstract}

\section{Introduction}

In recent years there has been considerable interest in understanding the dynamics of flows with localized vortex structures such as filaments and rings. Despite considerable progress, relatively little is known about such dynamics in a full 3D setting. The present work is aimed at studying general disturbances to axisymmetric vortex rings with (and without) swirl. Such rings are important as examples of exact solutions to the 3D Euler equations, with the vorticity localized inside a toroidal vortex core. We have computed several vortex rings with swirl numerically, and compared direct numerical simulations of the time-dependent behavior of perturbations to the rings with analytical predictions of instabilities. These predictions are based on a geometric optics, or short-wave approximation, in contrast with much analysis in which it is the low modes that are of primary interest. Further details are presented in [16].

The structure of vortex rings is governed by a quasi-linear elliptic equation for the streamfunction subject to appropriate regularity conditions. The main difficulty in finding exact rings stems from the fact that the location of the vorticity core boundary is not known a priori. Individual vortex rings are characterized by the profile functions describing the dependence of the azimuthal velocity and vorticity on the streamfunction as well as certain global constraints determining the location of the core boundary.

Until very recently, most analyses of vortex rings were concerned with the behavior in the thin ring limit, i.e., core radius small compared to outer radius, and with the structure of rings without 
swirl. For a recent review of vortex rings, see [20]. For the theory of thin rings, see [19]. The stability of thin rings has been studied by various authors, including Moore, Saffman, and Widnall. The exact theory of vortex rings without swirl has been developed by Norbury, Fraenkel, and others. Knio and Ghoniem [11] computed instabilities to vortex rings without swirl, using vortex methods, and compared the results with the thin ring theory. For more extensive references, see [20] and $[16]$.

Vortex rings with swirl have attracted considerably less attention than their no-swirl counterparts, although they represent a more general class of steady solutions of the Euler equations and play an important role in nature. Moffatt [17] developed an approach to the description of vortex rings with swirl based on their analogy with toroidal equilibrium configurations known in magnetohydrodynamics. Turkington [22] proved the existence of exact solutions, using a different approach based on the variational formulation of the corresponding quasi-linear boundary value problem. Eydeland and Turkington developed a numerical algorithm based on this approach, allowing one to obtain vortex rings corresponding to a large variety of profile functions and normalization constraints. This family includes the limiting cases of vortex rings without swirl, for which the vorticity and the velocity are orthogonal, and also the so-called Beltrami rings, for which they are parallel.

Vortex rings with swirl are natural objects of numerical study using vortex methods, because the initial distribution of vorticity is localized in a finite domain and can be chosen to be smooth. (Recall that Hill's vortex has a jump in the vorticity at the boundary of the core.) Such a study is useful for two reasons. First, it helps to assess the validity of 3D vortex methods, and, second, it sheds light on the dynamics of the rings as such. However, to draw sensible conclusions from such a study it is necessary to find the initial distribution of vorticity inside the vortex core with sufficient accuracy and to analyze the stability properties of the corresponding ring in advance. The latter is important to discriminate between the actual dynamics and numerical errors. Such a study was undertaken by Beale, Eydeland and Turkington [4] a few years ago. However, at that time numerical algorithms for generating exact vortex rings were not sufficiently accurate for this purpose, while the stability theory for vortex rings with swirl was virtually nonexistent. In addition, the ring used as a test in [4] was not well resolved and not very stable, so that the results were rather inconclusive.

Our objective is a more definitive study of time-dependent behavior than in [4], with numerics supported by comparison with analytic predictions. There are three compelling reasons for doing this. First, we have considerably improved the equilibrium solver by incorporating into the scheme appropriate matching conditions on the boundary of the computational domain. As a result, we are able to find the vorticity distribution inside the vortex core with very high accuracy. Second, we can apply a novel theory of stability of vortex rings, based on the geometrical optics approach, which was proposed recently by Lifschitz and Hameiri [12-15]. In this framework we can describe the initial behavior of highly localized perturbations to a given vortex ring analytically. We emphasize that the perturbation growth is similar in many respects to the transient growth observed in other settings. Third, we have developed a modified vortex method which is particularly well suited for studying the dynamics of the vortex rings (cf. [21]). We explicitly take into account the presence of the steady background flow in the problem, and also use the fast summation technique.

The asymptotic analysis used here, while familiar in other settings such as linear wave motion, has only recently been developed for applications such as inviscid fluid flow. Approximate equations are found for disturbances characterized by a rapid spatial oscillation. The eikonal equation, determining the phase function, has as its characteristics the particle paths of the underlying flow field. The disturbance is then described by ordinary differential equations along these particle paths for a wave vector and amplitude. Once an exact vortex ring has been computed numerically by the method referred to above, these ordinary differential equations can be solved numerically for 
various choices of particle paths. In this way the maximum rate of growth for short-wave disturbances can be estimated at various locations in the ring. The growth rates obtained depend on the streamfunction, which, roughly speaking, measures the distance from the center of the core of the ring.

The disturbances predicted by the short-wave analysis have been called "broad-band" instabilities [2], since they apply to high wavenumbers, rather than particular ones. A study similar in spirit to this one was carried out by Bayly [2]. He considered instabilities to quasi-two-dimensional steady flow, i.e., a periodic 2D flow with a third component added. He compared short-wave asymptotics with numerical solutions obtained using a spectral method.

In the direct simulations using the vortex method, we study the solutions of the 3D Euler equations corresponding to initial data with small deviation from the numerically computed equilibrium vorticity distributions and reproduce the dynamics (or lack thereof) of such solutions. We also consider initial data deviating by an appropriately chosen perturbation from the equilibrium vorticity distributions and explore the relationship between predictions of the stability theory and the numerical experiments. Typically, the growth rates observed from vortex method simulations are about half the maximum growth rate predicted by the geometric optics theory. Since we have not chosen the initial disturbances in any special way, we could not expect closer agreement without more detailed comparison of the two approaches. The results indicate that the version of the vortex method used in our analysis gives an adequate picture of the vortex dynamics for a relatively long time (on the order of a turn-around period in the poloidal plane), and that the solutions are in reasonable agreement with predictions of the geometrical optics stability theory. We hope that this combined study will improve understanding of the full stability properties of vortex rings, which are unknown even for rings without swirl.

\section{Vortex rings with swirl}

The vortex rings of interest here are exact solutions of the Euler equations of inviscid, incompressible flow. Their vorticity is limited to a bounded region; the vorticity can be chosen to be fairly smooth as a function of the spatial variables. The flow is axisymmetric and independent of time except for translation along the axis.

Vortex rings are most naturally expressed in cylindrical coordinates $(r, \theta, z)$, with the $z$-axis as the axis of symmetry. Both the velocity and vorticity fields are functions of $(r, z)$; they can have any direction if swirl is included. The $\theta$-component of the velocity is called the swirl velocity. The vortex core is a toroidal region symmetric about the $z$-axis. In vortex rings without swirl, the particles move along two-dimensional paths, and the flow has much of the character of two-dimensional flow. In particular, the vorticity can be expressed in terms of a scalar field which is conserved. Rings without swirl have been studied extensively, and the stability characteristics of thin rings are understood reasonably well. Exact vortex rings with swirl have more general behavior, and less is known about their properties. Within the vortex core of such rings the particles move in helices, and the flow has distinct three-dimensional features. In this sense, vortex rings with swirl serve better to illustrate $3 \mathrm{D}$ flow than those without.

We begin by treating vortex rings as steady solutions of the Euler equations:

$$
\begin{aligned}
\frac{\partial \mathbf{u}}{\partial t}+\mathbf{u} \cdot \nabla \mathbf{u} & =-\nabla p \\
\nabla \cdot \mathbf{u} & =0
\end{aligned}
$$

where $\mathbf{u}$ is the velocity and $p$ is the pressure. We rewrite the steady equations in modified cylindrical coordinates $(y, \theta, z)$, with $y=r^{2} / 2$. It can be shown that there is a Stokes streamfunction $\psi=$ 
$\psi(y, z)$ such that the velocity $\mathbf{u}$ and the vorticity $\boldsymbol{\omega}$ have the form

$$
\begin{gathered}
\mathbf{u}=u^{y} \hat{e}_{y}+u^{\theta} \hat{e}_{\theta}+u^{z} \hat{e}_{z}=-\frac{1}{\sqrt{2 y}} \psi_{z} \hat{e}_{y}+u^{\theta} \hat{e}_{\theta}+\psi_{y} \hat{e}_{z}, \\
\boldsymbol{\omega}=\omega^{y} \hat{e}_{y}+\omega^{\theta} \hat{e}_{\theta}+\omega^{z} \hat{e}_{z}=-u_{z}^{\theta} \hat{e}_{y}-\frac{1}{\sqrt{2 y}} \mathcal{L} \psi \hat{e}_{\theta}+\left(\sqrt{2 y} u^{\theta}\right)_{y} \hat{e}_{z} .
\end{gathered}
$$

Here $\hat{e}_{y}, \hat{e}_{\theta}, \hat{e}_{z}$ are the orthonormal basis vectors in the cylindrical coordinate system, $\mathcal{L}$ is the linear elliptic operator

$$
\mathcal{L}=2 y \frac{\partial^{2}}{\partial y^{2}}+\frac{\partial^{2}}{\partial z^{2}}
$$

and

$$
u^{\theta}=\frac{1}{\sqrt{2 y}} f(\psi), \quad \omega^{\theta}=-\sqrt{2 y} h^{\prime}(\psi)+\frac{1}{\sqrt{2 y}} f(\psi) f^{\prime}(\psi) .
$$

We have introduced arbitrary functions $f$ and $h$ of $\psi$, and $f^{\prime}=d f / d \psi, h^{\prime}=d h / d \psi$, representing the circulation around circles centered at the axis of symmetry and the Bernoulli head function, respectively. The streamfunction $\psi$ satisfies the equilibrium equation

$$
\mathcal{L} \psi=2 y h^{\prime}(\psi)-f(\psi) f^{\prime}(\psi)
$$

and the boundary conditions

$$
\psi=-\mu \quad \text { on } y=0 \text { and } \psi \rightarrow-(\mu+\nu y) \text { as }|(y, z)| \rightarrow \infty .
$$

We are looking for a steady solution with compactly supported vorticity which is embedded in the uniform flow. It is clear that $\psi$ is constant on the boundary of the vortex core, and we can assume that $f=0, h=h_{0}>0$ outside the core. The constants $\mu$ and $\nu$ represent the constant value of $\psi$ on the axis of symmetry and the uniform axial velocity at infinity, respectively. The values of $\mu$ and $\nu$ can be determined from two additional constraints expressing conservation of the meridional circulation $C$ and the axial component of linear impulse $P$, given below.

We choose the structure functions $f$ and $h$ to be

$$
f(\psi)=\sqrt{\frac{2 \alpha}{\zeta+1}} \psi_{+}^{(\zeta+1) / 2}, \quad h(\psi)=h_{0}-\frac{\beta}{2(\zeta+1)} \psi_{+}^{\zeta+1},
$$

where $\psi_{+}=\max (\psi, 0)$, and $h_{0}, \alpha, \beta, \zeta$ are arbitrary positive constants. (The constant $h_{0}$ has no effect.) After substitution in (5), the equilibrium problem reduces to the equation:

$$
\mathcal{L} \psi=-(\alpha+\beta y) \psi_{+}^{\zeta},
$$

subject to the constraints

$$
\begin{gathered}
\iint \frac{1}{y}(\alpha+\beta y) \psi_{+}^{\zeta} d y d z=1 \\
\iint(\alpha+\beta y) \psi_{+}^{\zeta} d y d z=1
\end{gathered}
$$

and the inhomogeneous boundary conditions (6). Here we assume that $P=1$ and $C=1$. This normalization guarantees that the linear size of the vorticity core is of order unity. The quantities to be determined are the streamfunction $\psi$ and the constants $\mu, \nu$. Turkington [22] proved via a variational method the existence of a solution to this problem in the half-plane. Eydeland and 
Turkington [9] extended this proof to produce an iterative numerical method for solving the problem (8), (9), (10) in a finite computational domain $R=\{(y, z) \mid 0 \leq y \leq Y,-Z \leq z \leq Z\}$ with the mixed Dirichlet-Neumann boundary conditions. They assumed that the size of the domain $R$ is so large that the numerical boundary does not have an appreciable effect on the solution inside the vorticity core.

Our approach to computing solutions is also based on a reduction of the problem in the halfspace to a problem in a finite computational domain $R$. However, we replace the mixed Dirichlet-

Neumann boundary conditions by the following nonreflective (and nonlocal) conditions on the boundary of $R$ :

$$
\psi(y, \pm Z)=\phi(y, \pm Z)-\mu-\nu y, \quad \psi(0, z)=\mu, \quad \psi(Y, z)=\phi(Y, z)-\mu-\nu Y,
$$

where

$$
\phi(y, z)=-\iint \mathcal{G}(y, \bar{y}, z, \bar{z})(\alpha+\beta \bar{y}) \psi_{+}^{\zeta} d \bar{y} d \bar{z} .
$$

Here $\mathcal{G}$ is the Green's function for the operator $\mathcal{L}$ in the free space. It is clear that $\phi$ represents the exact solution on the computational boundary of the half-space problem for $\psi+\mu+\nu y$. Conditions (11) guarantee that the streamfunctions inside the computational domain can be smoothly extended to infinity. Accordingly, we can restrict ourselves to a much smaller computational domain provided that it contains the vorticity core. This is a crucial step towards improving the accuracy of the numerical method. The actual iterative scheme is an appropriately modified version of the one used by Eydeland and Turkington. It is given explicitly in [16].

A variety of vortex rings can be generated by the method discussed here with characteristics governed by the three parameters $\alpha, \beta$, and $\zeta$. In particular, $\zeta$ controls the smoothness of the streamfunction $\psi$ on the boundary of the vortex core. Larger values of $\zeta$ produce smoother matching at the boundary. In principle, it is desirable to make $\psi$ as smooth as possible to avoid numerical errors. However, requiring too much smoothness at the boundary results in a streamfunction with a complicated structure and a vortex ring which is highly unstable. A particular example of this can be seen in [4] where an exceptionally unstable ring, with $\zeta=3$, was used as a test problem. Setting $\zeta=1.5$ seems to be a reasonable compromise. Once $\zeta$ is fixed, we are left with a two parameter family of vortex rings depending on $\alpha$ and $\beta$. The special case with $\alpha=0$ produces rings without swirl, for which the velocity and vorticity are perpendicular. On the other hand, setting $\beta=0$, produces the so-called Beltrami rings which have parallel velocity and vorticity.

\section{Analysis of local instabilities}

Once a vortex ring is constructed by the method of the previous section, we have available an exact solution of the Euler equations of incompressible, inviscid flow. We can predict the behavior of disturbances to this exact solution by numerical simulations, as described subsequently, or by analytical treatment of instabilities. Although there has been considerable research on the stability of thin rings, little is known about the stability of exact rings with swirl. In this section we discuss the stability of general vortex rings using a WKB type method developed by Lifschitz and Hameiri [12, 13, 14, 15], valid for short wavelengths and small amplitude perturbations. Once the growth rates of these instabilities have been calculated, we can compare them with the results of time-dependent numerical simulations. This gives us an opportunity to determine the general applicability of the WKB method.

In the WKB approximation to inviscid flow, short wavelength instabilities are described in terms of localized wave envelopes characterized by the phase $\Phi$ and the amplitude. These envelopes move 
along with the particles in the fluid flow, and their behavior is governed by a system of characteristic equations along particle trajectories [12]. This system consists of the trajectory equation for fluid particles, eikonal equation for the wave vector and transport equation for the velocity amplitude. The stability properties of the flow are determined by the growth of solutions to this system of ordinary differential equations.

We begin by assuming a linear disturbance in the form $\mathbf{u}_{1}(t, \mathbf{x}) \exp (i \Phi(t, \mathbf{x}) / \epsilon)$ plus lower order terms. If $\mathbf{x}_{p}$ is a particular particle location, moving with the flow, we define $\mathbf{a}(t)=\mathbf{u}_{1}\left(t, \mathbf{x}_{p}\right)$ and $\mathbf{k}(t)=\nabla \Phi\left(t, \mathbf{x}_{p}\right)$. The characteristic equations turn out to be

$$
\begin{gathered}
\frac{d \mathbf{x}}{d t}=\mathbf{u}_{s}, \\
\frac{d \mathbf{k}}{d t}=-\nabla \mathbf{u}_{s} \cdot \mathbf{k}, \\
\frac{d \mathbf{a}}{d t}=-\mathbf{a} \cdot \nabla \mathbf{u}_{s}+2 \frac{\mathbf{a} \cdot \nabla \mathbf{u}_{s} \cdot \mathbf{k}}{|\mathbf{k}|^{2}} \mathbf{k},
\end{gathered}
$$

supplemented by an initial condition

$$
\mathbf{x}(0)=\mathbf{x}_{0}, \quad \mathbf{k}(0)=\mathbf{k}_{0}, \quad \mathbf{a}(0)=\mathbf{a}_{0}
$$

and the incompressibility condition

$$
\mathbf{a} \cdot \mathbf{k}=0
$$

which is compatible with the characteristic equations. The trajectory equation (13) determines the streamline passing through $\mathbf{x}_{0}$. Here $\mathbf{u}_{s}$ means the velocity in the undisturbed vortex ring. The eikonal equation (14) determines the evolution of the local wave vector $\mathbf{k}$ along this streamline, and similarly the transport equation (15) determines the evolution of the amplitude $\mathbf{a}$.

Lifschitz and Hameiri [15] proved that a vortex ring is unstable in the velocity norm if for certain initial data $\mathbf{x}_{0}, \mathbf{k}_{0}, \mathbf{a}_{0}$ such that $\mathbf{a}_{0} \cdot \mathbf{k}=0$, the corresponding amplitude $\mathbf{a}(t)$ grows in time without a bound. This observation allows us to analyze the stability of a given vortex by numerically solving the system of ordinary differential equations (13), (14), (15) along streamlines of this ring. Note that the system of characteristic equations has a triangular structure, i.e., the trajectory depends only on $\mathbf{x}_{0}$; the wave vector depends on both $\mathbf{x}_{0}$ and $\mathbf{k}_{0}$; and finally, the amplitude depends on $\mathbf{x}_{0}, \mathbf{k}_{0}$, and $\mathbf{a}_{0}$. It is therefore natural to solve them in succession. Using the specific structure of the vortex ring, we can write the equations more explicitly in terms of the streamfunction $\psi$; see $[16]$.

We start with Eq. 13 describing helical trajectories of the fluid particles. Projections of these trajectories onto the $y, z$-plane are closed; we denote the period of rotation by $T$. Next we consider Eq. 14 for the wave vector. It can be shown that this equation has a special structure which enables one to find a fundamental system of solutions explicitly. Lifschitz and Hameiri [15] constructed a fundamental system of solutions $\mathbf{K}_{i}, i=1,2,3$, two of which (say $\mathbf{K}_{1}$ and $\mathbf{K}_{2}$ ) are periodic with period $T$, and the third one, $\mathbf{K}_{3}$ grows linearly in time and becomes asymptotically proportional to $t \nabla \psi$. Thus Eq. 14 has a two-parameter family of periodic solutions, while all other solutions grow linearly in time.

Finally we consider the transport equation (15) for the amplitude and denote by $\mathcal{F}$ the fundamental matrix solution of this equation, i.e. solution satisfying the initial conditions $\mathcal{F}(0)=\mathcal{I}$. In order to satisfy the incompressibility condition (17) we need to project $\mathcal{F}$ onto the subspace orthogonal to $\mathbf{k}$, i.e., to consider the projected fundamental matrix $\mathcal{F}_{\pi}=\mathcal{F}\left(\mathcal{I}-\mathcal{P}_{\mathbf{k}_{0}}\right)$. The matrix $\mathcal{F}_{\pi}$ depends on $\mathbf{x}_{0}$ and $\mathbf{k}_{0}$. For the purpose of comparing the analytical and numerical results, we 
have to study the finite-time behavior of solutions of the transport equation on a certain finite time interval $0 \leq t \leq t^{*}$, rather than the large time limit. The solutions are determined by the behavior of the projected fundamental solution $\mathcal{F}_{\pi}$. We define the finite-time growth rate by the formula

$$
\sigma_{t^{*}}=\frac{1}{2 t^{*}} \log \left\|\mathcal{F}_{\pi}^{t r}\left(t^{*}\right) \mathcal{F}_{\pi}\left(t^{*}\right)\right\|,
$$

where $\mathcal{F}_{\pi}^{t r}$ is the transpose of $\mathcal{F}_{\pi}$. It depends on $\mathbf{x}_{0}$ and the direction of $\mathbf{k}_{0}$. The value of $\sigma_{t^{*}}$, maximized over all initial vectors $\mathbf{k}_{0}$, gives the largest finite time growth rate associated with the point $\mathbf{x}_{0}$. In order to obtain the growth rate which characterizes a stream surface, we average the maximized $\sigma_{t^{*}}$ over various initial points belonging to the same stream surface. It is this growth rate that we are going to compare with the one observed in the numerical experiments in Section 4.

With these general ideas in mind, we now describe the numerical procedure used to obtain specific values of the growth rates for various stream surfaces. First, we use the method described in the previous section to generate a vortex ring with specific choices of the parameters $\alpha, \beta, \zeta$. In this computation we obtain the values of $\psi$ on the grid points inside the computational rectangle $R$. Then we extrapolate the values of $\psi$ onto the intermediate points using the bi-cubic splines. In this way we obtain analytical expressions for $\psi$ and its partial derivatives everywhere in $R$. Using these expressions we solve Eq. 13 and determine the trajectory passing through the point $\mathbf{x}_{0}$. Next, we solve Eq. 14 and find three linearly independent solutions $\mathbf{K}_{i}, i=1,2,3$ of the eikonal equation. We consider a two-parameter family of $\mathbf{k}_{0}$ of the form

$$
\mathbf{k}_{0}=\cos \mu \sin \nu \mathbf{K}_{1}(0)+\sin \mu \sin \nu \mathbf{K}_{2}(0)+\cos \nu \mathbf{K}_{3}(0)
$$

and compute the corresponding projected fundamental solution for Eq. 15. We then scan the $\mu, \nu$ parameter space to find the maximum value of the finite-term growth rate $\sigma_{t^{*}}$ with respect to $\mathbf{k}_{0}$, using formula (18). Finally, we average this along the stream surface. The resulting values for representative surfaces are reported for several rings in Section 4.

\section{Vortex method calculations}

We compute numerical solutions of the time-dependent Euler equations using a three-dimensional vortex method. Such methods have been used extensively since the early work of Chorin and Leonard. Several versions are in use, but all are closely related and based on the Biot-Savart law,

$$
\mathbf{u}(\mathbf{x}, t)=\int K\left(\mathbf{x}-\mathbf{x}^{\prime}, t\right) \boldsymbol{\omega}\left(\mathbf{x}^{\prime}, t\right) d \mathbf{x}^{\prime}, \quad K(\mathbf{x})=-\frac{\mathbf{x}}{4 \pi r^{3}} \times
$$

which expresses velocity $\mathbf{u}$ in terms of vorticity $\boldsymbol{\omega}$. The vortex method reduces the Euler equations to ordinary differential equations for the position and vorticity of a representative collection of Lagrangian particles. The version used here is essentially the one which was introduced in [1] and proved to converge in [3] and [7]. See also [5], [10], [18]. The calculations reported here exploit fourfold symmetry and also use a fast summation algorithm of Buttke [6], related to the Greengard-Rokhlin algorithm, to reduce the computational load. In addition the method is modified specifically to calculate perturbations and eliminate the steady solution from the computation.

The evolution of the flow field is represented by a collection of particles moving with the fluid. Their locations $\mathbf{x}_{j}(t)$ and corresponding values of the vorticity vector $\boldsymbol{\omega}_{j}(t)$ are calculated at each time. The velocity field can then be approximated from the Biot-Savart law as

$$
\mathbf{u}^{h}(\mathbf{x}, t)=\sum_{j} K_{\delta}\left(\mathbf{x}-\mathbf{x}_{j}\right) \boldsymbol{\omega}_{j} h^{3}
$$


where $K_{\delta}$ is a version of the kernel of (20), smoothed by a blob function. The particle locations $\mathbf{x}_{j}(t)$ move according to this computed velocity. The vorticity evolution, given by the equation

$$
\frac{D \boldsymbol{\omega}}{D t}=\boldsymbol{\omega} \cdot \nabla \mathbf{u}
$$

can be approximated along the particle paths by

$$
\frac{d \boldsymbol{\omega}_{i}}{d t}=\boldsymbol{\omega}_{i} \cdot \nabla \mathbf{u}_{i}^{h}
$$

The derivative $\nabla \mathbf{u}_{i}^{h}$ can be obtained by a centered difference quotient after observing that the dot product reduces to a directional derivative. The particles are introduced initially on a square grid of size $h$ covering the vortex core. The blob function used here is a sixth degree radial polynomial within unit radius and zero otherwise. It is chosen to be continuous and fourth order accurate (see [16] or [21]). While there are other possible evolution equations for the vorticity, the one presented here is conceptually simple, is straightforward to implement, and seems to produce the best numerical results (see [4] for a discussion). Finally, the resulting ODE's are discretized in time by the fourth order Runge-Kutta method.

Next we introduce a modification which allows us to eliminate the steady part of the flow in the vortex ring calculation and concentrate on the evolution of perturbations. No new error is introduced by doing this. We impose an initial velocity perturbation $\mathbf{u}_{0}(\mathbf{x})$ on the steady velocity field of an exact ring, $\mathbf{u}_{s}(\mathbf{x})$. We want to find $\mathbf{u}(\mathbf{x}, t)$ so that $\mathbf{u}(\mathbf{x}, 0)=\mathbf{u}_{0}(\mathbf{x})$ and $\mathbf{u}_{s}(\mathbf{x})+\mathbf{u}(\mathbf{x}, t)$ is a solution to the Euler equations (1). The corresponding steady vorticity and vorticity perturbation are $\boldsymbol{\omega}_{s}(\mathbf{x})=\nabla \times \mathbf{u}_{s}$ and $\boldsymbol{\omega}(\mathbf{x}, t)=\nabla \times \mathbf{u}$ respectively. Since $\mathbf{u}_{s}+\mathbf{u}$ satisfies the Euler equations and $\boldsymbol{\omega}_{s}+\boldsymbol{\omega}=\nabla \times\left(\mathbf{u}_{s}+\mathbf{u}\right)$, then $\boldsymbol{\omega}_{s}+\boldsymbol{\omega}$ and $\mathbf{u}_{s}+\mathbf{u}$ must satisfy (22) and (20), leading to

$$
\begin{gathered}
\frac{D \boldsymbol{\omega}}{D t}=\left(\boldsymbol{\omega}_{s}+\boldsymbol{\omega}\right) \cdot \nabla \mathbf{u}+\boldsymbol{\omega} \cdot \nabla \mathbf{u}_{s}-\mathbf{u} \cdot \nabla \boldsymbol{\omega}_{s}, \\
\mathbf{u}(x, t)=\int K\left(\mathbf{x}-\mathbf{x}^{\prime}\right) \boldsymbol{\omega}\left(\mathbf{x}^{\prime}, t\right) d \mathbf{x}^{\prime}
\end{gathered}
$$

where $D \boldsymbol{\omega} / D t=\left(\mathbf{u}_{s}+\mathbf{u}\right) \cdot \nabla \boldsymbol{\omega}$ is the material derivative. We then discretize the problem to a system of evolution equations,

$$
\begin{gathered}
\frac{d \mathbf{x}_{i}}{d t}=\mathbf{u}_{s}\left(\mathbf{x}_{i}\right)+\sum_{j} K_{\delta}\left(\mathbf{x}_{i}-\mathbf{x}_{j}\right) \boldsymbol{\omega}_{j} h^{3} \\
\frac{d \boldsymbol{\omega}_{i}}{d t}=\left(\boldsymbol{\omega}_{s}\left(\mathbf{x}_{i}\right)+\boldsymbol{\omega}_{i}\right) \cdot \nabla \mathbf{u}^{h}\left(\mathbf{x}_{i}, t\right)+\boldsymbol{\omega}_{i} \cdot \nabla \mathbf{u}_{s}\left(\mathbf{x}_{i}\right)-\mathbf{u}^{h}\left(\mathbf{x}_{i}, t\right) \cdot \nabla \boldsymbol{\omega}_{s}\left(\mathbf{x}_{i}\right),
\end{gathered}
$$

which we solve using the fourth order Runge-Kutta method. Since this procedure does not depend on vortex ring structure, it could be used to model the behavior of perturbations of any steady known solution of the Euler equations.

In studying the growth of instabilities to vortex rings, we can in principle vary the wavelength, shape, direction, and location of the initial disturbance, so that a thorough study would have to be quite extensive. Here we focus on the small amplitude perturbation with initial velocity

$$
\mathbf{u}_{0}=\Gamma(\Psi) \cos (20 \theta) \hat{r} .
$$


Table I: Vortex Ring Properties

\begin{tabular}{|c|c|c|c|c|c|c|}
\hline$\alpha$ & $\beta$ & $h$ & $n$ & prop. speed & $\psi_{\max }$ & $T_{\min }$ \\
\hline 20 & 20 & 0.10 & 3976 & 0.0637 & 0.101 & 14.0 \\
50 & 0 & 0.09 & 4315 & 0.0543 & 0.102 & 11.3 \\
50 & 20 & 0.08 & 4270 & 0.0671 & 0.103 & 7.76 \\
50 & 40 & 0.08 & 3289 & 0.0752 & 0.104 & 5.92 \\
80 & 20 & 0.08 & 2955 & 0.0702 & 0.104 & 5.35 \\
\hline
\end{tabular}

and corresponding curl

$$
\begin{gathered}
\boldsymbol{\omega}_{0}=\frac{\partial}{\partial z}(\Gamma(\Psi) \cos (20 \theta)) \hat{\theta}-\frac{1}{r} \frac{\partial}{\partial \theta}(\Gamma(\Psi) \cos (20 \theta)) \hat{z}= \\
\frac{d \Gamma(\Psi)}{d \Psi} \frac{\partial \Psi}{\partial z} \cos (20 \theta) \hat{\theta}+\frac{20}{r} \Gamma(\Psi) \sin (20 \theta) \hat{z}
\end{gathered}
$$

where $\Gamma$ is a smooth cut-off function in $\Psi$ with amplitude on the order of 0.003 . Using the modified vortex method described above, we calculate the evolution of this perturbation for five vortex rings. In order to compare these results with the analytical work discussed earlier, we need to compute localized growth rates which are compatible in type with the predictions. We examine perturbations by following the evolution in time of special tracking particles introduced near the level curves of $\Psi$ defined by $\Psi_{i}=i \Psi_{\max } / 10$ for $1 \leq i \leq 10$. While the velocities and positions of these tracking particles are computed, they are not used in the vortex method calculation. A relative $L^{2}$ velocity deviation from the steady ring is then computed and can be compared with the analytical results.

The five vortex rings we consider here are produced by varying the parameters $\alpha$ and $\beta$ in the structure functions (7). For all of these rings the parameter $\zeta=1.5$. The perturbed solutions are calculated in time steps of $\Delta t=0.2$ until $t=9.8$, which is roughly the period of a particle near the center of the ring moving in the poloidal cross section; smaller time steps did not affect the results. Fast summation and fourfold symmetry are used in all calculations. Further information about these rings is contained in Table I. In this table, $h$ is the mesh size and $n$ is the number of vortex elements in $1 / 4$ of the vortex ring.

Average predicted and observed growth rates between $t=0$ and $t=9.8$ are listed in Table II. The predicted rates are computed from the geometric optics approach by the procedure described in the previous section. The index $i$ in the table refers to the stream surface with $\Psi=\Psi_{i}$. Even though the growth rate predictions are made for short wavelength local instabilities, they do seem to be relatively good predictors of the overall stability of a vortex ring. Those with higher predicted growth rates have higher observed growth rates. Notice that the predicted maximum growth rate is generally 2 to 3 times larger than the observed growth rate, except in the first case. Finally, notice that the rings become less stable as $\alpha$ increases while the effect of $\beta$ on stability is unclear.

It is important to remember that the geometric optics analysis predicts a maximum possible growth rate, while the vortex method calculates the growth rate for a particular perturbation. A particular solution typically will have a growth rate somewhat less than the predicted maximum. A closer comparison might be obtained by specializing the geometric optics prediction to the particular perturbation used in the vortex method calculation. The WKB method only describes the growth of short wavelength local instabilities. Longer wavelength perturbations and nonlocal effects may also be important in the evolution of a vortex ring. Notice that within a particular vortex ring in Table II, the predicted growth rates vary more than the observed growth rates. We may expect that 
Table II: Average Predicted and Observed Growth Rates

\begin{tabular}{|c|c|c|c|c|c|c|c|c|c|c|}
\hline \multirow{2}{*}{$i$} & \multicolumn{2}{|c|}{$\alpha=20, \beta=20$} & \multicolumn{2}{c|}{$\alpha=50, \beta=0$} & \multicolumn{2}{c|}{$\alpha=50, \beta=20$} & \multicolumn{2}{c|}{$\alpha=50, \beta=40$} & \multicolumn{2}{c|}{$\alpha=80, \beta=20$} \\
\cline { 2 - 11 } & Pred. & Obs. & Pred. & Obs. & Pred. & Obs. & Pred. & Obs. & Pred. & Obs. \\
\hline 1 & 0.180 & 0.0298 & 0.264 & 0.141 & 0.338 & 0.148 & 0.394 & 0.150 & 0.470 & 0.276 \\
2 & 0.199 & 0.0287 & 0.291 & 0.142 & 0.370 & 0.153 & 0.425 & 0.153 & 0.522 & 0.280 \\
3 & 0.211 & 0.0228 & 0.308 & 0.157 & 0.387 & 0.155 & 0.437 & 0.156 & 0.559 & 0.281 \\
4 & 0.218 & 0.0274 & 0.317 & 0.154 & 0.396 & 0.151 & 0.438 & 0.158 & 0.572 & 0.284 \\
5 & 0.219 & 0.0315 & 0.319 & 0.166 & 0.396 & 0.151 & 0.420 & 0.163 & 0.558 & 0.290 \\
6 & 0.212 & 0.0344 & 0.314 & 0.160 & 0.384 & 0.151 & 0.387 & 0.168 & 0.530 & 0.297 \\
7 & 0.196 & 0.0377 & 0.302 & 0.150 & 0.351 & 0.147 & 0.347 & 0.167 & 0.495 & 0.302 \\
8 & 0.176 & 0.0380 & 0.276 & 0.132 & 0.292 & 0.137 & 0.308 & 0.156 & 0.445 & 0.298 \\
9 & 0.141 & 0.0288 & 0.225 & 0.115 & 0.199 & 0.119 & 0.255 & 0.134 & 0.350 & 0.280 \\
10 & 0.103 & 0.0158 & 0.139 & 0.102 & 0.111 & 0.076 & 0.130 & 0.097 & 0.169 & 0.249 \\
\hline
\end{tabular}

nonlocal effects force perturbations in different locations to grow at similar rates. In view of these qualifications, the predicted and observed growth rates seem to be in reasonably good agreement.

\section{Acknowledgements}

The first author was supported by N.S.F. Grant No. IRI-9224605, the second and third by N.S.F. Grant No. DMS-9102782 and the third by N.S.F. Grant No. DMS-9403402.

\section{References}

[1] Anderson, C. and Greengard, C., "On Vortex Methods," SIAM J. Numerical Analysis, 22, pp. 413-440, 1985.

[2] Bayly, B., "Computations of Broad-Band Instabilities in a Class of Closed-Streamline Flows," in Mathematical Aspects of Vortex Dynamics, edited by R. Caflisch, SIAM, Philadelphia, pp. 50-58, 1989.

[3] Beale, J.T., "A Convergent Three-Dimensional Vortex Method with Grid-Free Stretching," Math. Comp., 46, pp. 401-424 and S15-S20, 1986.

[4] Beale, J.T., Eydeland, A. and Turkington, B., "Numerical Test of 3D Vortex Methods Using a Vortex Ring with Swirl," in Vortex Dynamics and Vortex Methods, edited by C. Anderson and C. Greengard, Lec. in Appl. Math., 28, Amer. Math. Soc., Providence, pp. 1-9, 1991.

[5] Beale, J.T. and Majda, A., "High Order Accurate Vortex Methods with Explicit Velocity Kernels," J. Comp. Phys., 58, pp. 188-208, 1985.

[6] Buttke, T., "Fast Vortex Methods in Three Dimensions," in Vortex Dynamics and Vortex Methods, edited by C. Anderson and C. Greengard, Lec. in Appl. Math., 28, Amer. Math. Soc., Providence, pp. 51-66, 1991.

[7] Cottet, G.H., "On the Convergence of Vortex Methods in Two and Three Dimensions," Ann. Inst. H. Poincare, 5, pp. 227-285, 1988. 
[8] Eydeland, A. and Turkington, B., "A Computational Method of Solving Free-Boundary Problems in Vortex Dynamics," J. Comp. Phys., 78, pp. 194-214, 1988.

[9] Eydeland, A. and Turkington, B., "A Numerical Study of Vortex Rings with Swirl," preprint, 1988.

[10] Gustafasson, K. and Sethian, J., ed., Vortex Methods and Vortex Motion, SIAM, Philadelphia, 1991.

[11] Knio, O. and Ghoniem, A., "Numerical Study of a Three-Dimensional Vortex Method," $J$. Comp. Phys., 86, pp. 75-106, 1990.

[12] Lifschitz, A., "Short Wavelength Instabilities of Incompressible Three-Dimensional Flows and Generation of Vorticity," Physics Letters A, 157, pp. 481-487, 1991.

[13] Lifschitz, A., "On the Instability of Certain Motions of an Ideal Incompressible Fluid," Adv. Appl. Math., 15, pp. 404-436, 1994.

[14] Lifschitz, A. and Hameiri, E., "Local Stability Conditions in Fluid Dynamics," Phys. Fluids A, 3, pp. 2644-2651, 1991.

[15] Lifschitz, A. and Hameiri, E., "Localized Instabilities of Vortex Rings with Swirl," Comm. Pure Appl. Math. 46, pp. 1379-1408, 1993.

[16] Lifschitz, A., Suters, W.H. and Beale, J.T., "The onset of instability in exact vortex rings with swirl," to appear in J. Comp. Phys.

[17] Moffatt, H., "Generalised Vortex Rings with and without Swirl," Fluid Dyn. Res., 3, pp. 22-30, 1988.

[18] Puckett, E.G., "Vortex Methods: An Introduction and Survey of Selected Research Topics," in Incompressible Computational Fluid Dynamics, edited by M. Gunzburger and R. Nicolaides, Cambridge Press, Cambridge, pp. 335-407, 1993.

[19] Saffman, P., Vortex Dynamics, Cambridge Press, Cambridge, 1992.

[20] Shariff, K. and Leonard, A., "Vortex Rings," Ann. Rev. Fluid Mech., 24, pp. 235-279, 1992.

[21] Suters, W.H., A Numerical Study of the Instability of Vortex Rings with Swirl, Ph.D. Thesis, Duke Univ., 1994.

[22] Turkington, B., "Vortex Rings with Swirl: Axisymmetric Solutions of the Euler Equations with Nonzero Helicity," SIAM J. Math. Anal., 20, pp. 57-73, 1989. 